\title{
Introduction to special issue: Innovations for transport planning in China
}

\section{Zhibin Li}

Southeast University

lizhibin@seu.edu.cn

\section{Chuan Ding \\ Beihang University \\ cding@buaa.edu.cn}

\author{
Junfeng Jiao \\ The University of Texas at Austin \\ jjiao@austin.utexas.edu
}

\begin{abstract}
We planned this special issue in response to the new opportunities and innovations for urban transport planning in China all of which can help build the smart transportation systems of the future. In preparation for the special issue, we organized the 19th COTA International Conference of Transportation Professionals (CICTP2019) with the theme of "Transportation in China 2025" in July 2017 and recommended high-quality submissions to this special issue. In the end, we received a total of 24 valid papers. After the standard peer-review process, we accepted eight papers for the special issue, with three focusing on built environment and travel activity, three focusing on road network distribution, and two focusing on data-driven traffic modeling. The special issue also has a well-balanced research focus on different types of transport modes, with two papers on multi-modal transport, three on personal cars, two on public bikes, and one on pedestrians.
\end{abstract}

Keywords: Transport planning, travel behavior, dynamic traffic assignment, built environment, real-time route planning, connected vehicles

\section{Article history:}

Received: June 29, 2020

Available online: November 10, 2020

\section{$1 \quad$ Introduction}

China has been experiencing unprecedented urbanization over the past few decades and this trend is set to continue into the near future. Urban sprawl, the hallmark of US development, has begun to emerge in urban and suburban China. How to accommodate these new urban dwellers has become an imminent problem for all metropolitan areas in China. Moreover, with rising disposable income and continued economic reform, auto ownership has exploded in China. Auto-oriented policies have been increasingly favored in transportation decision-making across Chinese cities. Urban transport systems are facing significant challenges such as increased safety risks, congestion, and excessive air pollution and energy consumption. Given these societal changes, an important research question emerges: How can

Copyright 2020 Zhibin Li, Junfeng Jiao \& Chuan Ding

http://dx.doi.org/10.5198/jtlu.2020.1834

ISSN: 1938-7849 | Licensed under the Creative Commons Attribution - Noncommercial License 4.0

The Journal of Transport and Land Use is the official journal of the World Society for Transport and Land Use (WSTLUR) and is published and sponsored by the University of Minnesota Center for Transportation Studies. 
urban transport systems in Chinese cities be designed and planned to minimize the risks and maximize the benefits of rapid urbanization?

Many believe recent technological advances can be part of the solution. For example, novel sensing technologies are emerging, which are capable of tracking individual activities and movement trajectories on continuous temporal and spatial dimensions. Big Data provides opportunities to observe and model both individual motilities at disaggregated levels and collective travel patterns at aggregated levels in much finer geographic detail than ever before. Analyzing the new and emerging data requires novel analytical frameworks and methodologies. Furthermore, the nexus of transport planning and new technologies such as autonomous vehicles, new mobility options such as shared dockless bike systems could change the traffic system.

We planned this special issue in response to the new opportunities and innovations for urban transport planning in China all of which can help build the smart transportation systems of the future. In preparation for the special issue, we organized the 19th COTA International Conference of Transportation Professionals (CICTP2019) with the theme of "Transportation in China 2025" in July 2017 and recommended high-quality submissions to this special issue. In the end, we received a total of 24 valid papers. After the standard peer-review process, we accepted 8 papers for the special issue, with three focusing on built environment and travel activity, three focusing on road network distribution, and two focusing on data-driven traffic modeling. The special issue also has a well-balanced research focus on different types of transport modes, with two papers on multi-modal transport, three on personal cars, two on public bikes, and one on pedestrians.

Build environment in urban areas affects residents' travel activities as well as perceptions towards trips. Quantifying those impacts could help improve the built environments for a safe, comfort and efficient traffic system. Two papers in this special issue assess the interactions between built environment factors and travel activity patterns by adopting various modeling algorithms. Zhou, Li, Li, Wang, and Wang (2020) investigate the dynamic interactions between environment change and travel behaviors in old town fringes. Based on 5-year data, multiple logic models were developed to estimate the impacts of built environment, economic and social attributes on choice of travel modes. Lyu, Wu, Liu, and Liu (2020) explored the relationship between built environment and public bike ridership. Based on 22 million trip records from 1064 public bike docking stations, the multi-scale geographically weighted regression (MGWR) was adopted to capture the spatial heterogeneity effects among different regions. "Six-D" built environment factors are closely related to public bike use which includes density, diversity, design, destination accessibility, distance to transit, and demand management. Wu, Chen, Zhang, and Jiao (2020) explored the impact of street characteristics perceived safety of older pedestrians. 68 recruited elderly urban dwellers took a safety survey using 39 simulated streetscape images which contained diverse street characteristics. The ordinal logit regression results show that street interface type, elevation differences, footpath width, paver directions, isolation facility type, and vehicle traffic had impacts on older pedestrians' perceived safety.

Network traffic distribution is a crucial component in traffic planning in that it calculates the capacity of an urban road network and land use efficiency. Tan, Du, and Yu (2020) proposes a novel multiple-path gradient projection approach to solve the logit-based stochastic user equilibrium problem on road networks. A two-step solving method including moving along direction and project is proposed and three practical step size schemes, the fixed step size, the SRA scheme and the SAA strategy, are embedded. The results show that the proposed algorithm outperforms the original gradient projection method in terms of accuracy and computing speed. Tian, Chiu, Sun, and Chai (2020) propose a new approach based on existing massive vehicle trajectories to extract the interzonal travel impedance skim matrix in dynamic traffic assignment problems. Numerical tests on Shanghai expressway network and 
US metropolitan networks show that the proposed approach has the advantage of saving computing memory and time. Wang, P. (2020) develop a real-time regional route planning model based on information of signal time and phase collected by vehicle-to-x (V2X) communication. The results of numerical experiments show that the proposed algorithm achieved a shorter average travel time on optimal routes than traditional navigation routes.

Data-driven solutions could more accurately obtain travel activity patterns for traffic planning purposes than traditional model-driven solutions. Chen, Yang, and Xu (2020) applied the Logarith Gaussian Mixed Model (Log-GMM) for inferring trip modes with model phone signaling data. Based on data of 38,719,455 users in Shanghai, they performed the activity nodes recognition, travel time computation, and clustering with Log-GMM to infer trip modes and achieved an accuracy of $72.68 \%$. The proposed method has advantages of easy calculation and realization and has better performance in identifying bus, car, and subway, which can provide significant data reference for city transportation planning, construction, and operation. Usama, Shen, Zahoor, and Bao (2020) proposed an optimization model for minimizing total cost of rebalancing operation of free-floating bike sharing systems considering faulty bikes. A two-step procedure was executed which first gathers faulty bikes to a station by model traversing the shortest path and then rebalances bikes to obtain the optimal inventory at stations and faulty bikes at depots. Based on the bike distribution data, the formulated optimal model is calculated using CPLEX solver for various scenarios.

With a wide range of topics covered by the eight high-quality papers, we hope that this special issue will provide useful insights for the traffic planning innovations in China and contribute to the research efforts around the globe. 


\section{References}

Chen, X., Yang, C., \& Xu, X. (2020) Trip mode inference from mobile phone signaling data using logarithm Gaussian mixture model. Journal of Transport and Land Use, 13(1), 429-445.

Lyu, C., Wu, X., Liu, Y., \& Liu, Z. (2020) Exploring multi-scale spatial relationship between built environment and public bicycle ridership: A case study in Nanjing. Journal of Transport and Land Use, 13(1), 447-467.

Tan, H., Du, M., \& Yu, C-B. (2020) A multiple-path gradient projection method for solving the logitbased stochastic user equilibrium models. Journal of Transport and Land Use, 13(1), 539-558.

Tian, Y., Chiu, Y.-C., Sun, J., \& Chai, C. (2020) Sunsetting skim matrices: A trajectory-mining approach to derive travel time skim matrix in dynamic traffic assignment for activity-base model integration. Journal of Transport and Land Use, 13(1), 413-428.

Usama, M., Shen, Y., Zahoor, O., \& Bao, Q. (2020) Dockless bike-sharing system: Solving the problem of faulty bikes with simultaneous rebalancing operation. Journal of Transport and Land Use, 13(1), 491-515.

Wang, P. (2020) Real-time urban regional route planning model for connected vehicles based on V2X communication. Journal of Transport and Land Use, 13(1), 517-538.

Wu, H., Chen, Y., Zhang, Z., \& Jiao, J. (2020) The impact of street characteristics on perceived safety of walking among older adults in Shanghai, China. Journal of Transport and Land Use, 13(1), 469-490.

Zhou, W., Li, Q., Li, Z., Wang, N., \& Wang, Q. (2020) Dynamic interactions between spatial change and travel behavior variation in old town fringe. Journal of Transport and Land Use, 13(1), 559-584. 